\title{
Stephen Katz (ed.) (2018). Ageing in Everyday Life. Materialities and Embodiments. Bristol: Policy Press, 208 pp. ISBN 978-1447335917 (hardback)
}

\author{
REVIEWED by JOYCE WEIL*
}

Drawing from multiple bodies of theory and methodological approaches, Katz's edited volume achieves its mission: reminding us that ageing and older adults are often missing from both sociological discourses and discussions of intersectionality - that is, marginalisation rather than inclusion. This book shows the "materialities" and "embodiment" of ageing focusing on the complexity and influence of the material world upon constructed reality and lived experience of ageing and later life. Katz's selected authors put in context the work of Simmel, who writes that "the essence of aesthetic observation and interpretation comes from finding the typical in the unique" and that the beauty and "total meaning of the world as a whole radiates from every single point" (p. 7).

Structurally, Ageing in Everyday Life is part of the Ageing in a Global Context series. After Katz's (2018) introduction, the book has two sections, Materialities and Embodiments, each briefly introduced by Katz. The sections contain five chapters; an afterword ends the book. In Katz's text, Materialities refer to the "various places, technology, things, rhythms,

*Joyce Weil, Gerontology Program, College of Natural and Health Sciences, University of Northern Colorado, Greeley, CO, USA 
International Journal of Ageing and Later Life

designs, mobilities, and environments in which our experience of ageing is grounded and observable" (p. 1). Embodiments refer to "the orientation to bodily life as a site where dominant cultural narratives and forms of expertise about ageing gather" (p. 126).

In Part 1, "Materialities," Katz's introduction raises the issue of transience and mobility with material possessions as a constant amongst places. Items taken along are mutable ways to track identity. Ekerdt's chapter, "Things and possessions," uses United States Health and Retirement Survey (HRS) data to illustrate the material convoy, items accumulated throughout the lifecourse. Collecting things helps form identity, but items can be a weight and need to be managed. Ekerdt uses HRS data to show that divestment of things is not typical with age. Next steps for research could show how meaning/attachments to things may change at different points in one's lifecourse (i.e. something once valued highly may be discarded at another age).

Braedley's "Reinventing the nursing home" uses photovoice and ethnography to explain how ruling metaphors apply the design of other places, such as a hospital, hotel or home, to nursing-home design. The hospital metaphor portrays skilled care as sterile and with a medical lens. The home metaphor is domestic but sanitised, and the hotel metaphor uses tropes such as chandeliers, flowers and chef-based buffet. Ruling metaphors are problematic because they proscribe the material conditions, organisation infrastructure and relationships for nursing-home residents - controlling residents' lives and limiting innovation in nursing-home concept development. Ruling metaphors are like shadow institutions that never reach the fantasy they seek.

Gavin and Grenier's "The every-breaking wave of everyday life" applies nonrepresentational theory (NRT) to older peoples' movement in everyday life. They challenge long-held views of movement as biomechanics akin to active ageing. They replace it with concepts based upon the rhythm of everyday life. For example, momentum looks at how past behaviours positively (being swept up) or negatively (being lost) persist. Infectiousness is not contagion, but feelings passed between people that can change peoples' lives. Encountering is a favourable interruption each day to create one's world. This NRT approach suggests we must develop new methodologies and lenses to see the world of movement for older adults. 
Ageing in Everyday Life. Book review

Chivers' "What's exotic about The Best Exotic Marigold Hotel?" looks at the silver screen as a vehicle for perpetuating ageism. She shows how a hotel in India for retired English people serves as a colonial outpost substitute. The English older adults are depicted as having know-how, while the South-Asian older adults are portrayed as child-like. Chivers describes how the film portrays ageism hidden behind an exotic setting with complicated images of India as the mystical east, exotic and commonplace at the same time.

Rozanova, Wada and Hurd Clarke's "Between ageing and ageism" uses Goffman's spoiled identities to the evaluate older adults' online dating presence. They found that these dating sites are youth-centric and seen as so novel that they were covered in news media. Through ethnographic analysis, the authors found that such sites define the "good" ageing body and normalise sex and dating in later life but also pose risks of too quickly forming emotional intimacy. The authors question whether online dating and romance are the electronic version of successful ageing.

In Part II, Embodiments, Katz's introduction raises the crucial point: even in embodiment work, older adults' bodies are often absent. Sandberg's "Closer to touch" examines older men's sexuality through their own diary entries, moving the discussion of sexuality beyond solely potency. This true embodied approach produces views often unexplored older men missing intimacy, differences between physical and emotional intimacy becoming clearer, an increased need for skin-skin contact, and the impact of loss and illness on intimacy. All themes pointed out the real humanity of touch.

Gish, Grenier and Vrkljan's "Ageing bodies, driving and change" reframes how we look at fit between new Advanced Vehicle Technologies (AVT; e.g. dashboard GPS, backup sensors) and older bodies. Their interviews with older drivers explore the human-machine interface. Some older adults described AVT making them feel disconnected from the driving experience with former driving habits (looking over one's shoulder) not needed anymore. Others described benefits of AVT. The researchers called for car manufacturers to consider real human factors in ageing when making changes.

Kontos and Grigorovich's "Dancing with dementia" finds dance helps assert personhood. Through their observational work in a skilled-care 
International Journal of Ageing and Later Life

facility, the authors found dance rituals maintained identity of Hassidic residents. Dance expressed passion during prayer in religious services. Dance happened as unstructured, serendipitous movement in hallways (e.g. a woman in a wheelchair freely moving her arms and dancing for joy). Dance is an embodied selfhood raising body consciousness and revealing cultural embodiments - especially for those with dementia. The researchers call for dance programmes to utilise dance's therapeutic effects and build upon human capacity, not loss.

Twigg's "Why clothes matter" explores how clothes are both an intimate experience and a public expression. Clothes reflect how society constructs and assesses successful ageing by dress. Twigg studied older women in the UK and found ideas of lost style and lost pleasure in dress, but older women also felt they shed the "pinney" of their grandmothers and dressed much better at the same age. Women did not want to dress "old" but noted added surveillance of dressing for older women (e.g. Is the outfit the new style? Hides the ageing body?). Clothes, being the environment closest in, act as a vehicle of remembrance, with the clothed body becoming a site for the politics of ageing to play out.

"Our Fitbits, our (ageing) selves," by Marshall, reviews how devices regulate everyday life of older adults via the quantification of movement. While marketed as ways to keep older adults young and independent, these devices confirm more than steps. They influence identity and "successful" ageing regulation, making older adults "live by the numbers" (p. 197). Marshall deftly uses narrative, such as the woman who compares her reaction to her device's vibrations to a "pigeon getting its pellet" (p. 203). Marshall calls adults to challenge the dictation of exercise as the only definition of health and resist using movement to colonise the ageing body.

Sawchuk's "Afterword" sums up themes with Butler's apt quote: "We cannot talk about a body without knowing what supports the body and what its relation to that support - or lack of support - might be" (p. 223).

The flow and placement of chapters ground ethereal materialities and embodiment in the words of older adults. The overall text shifts the older body as object of biomedical and other surveillance and regulation to a site (and struggle) for identity expression. While Katz choses chapters 
addressing materialities and embodiment concepts, the materialitiesembodiment pair of terms is historically intertwined with complex and interrelated relationships, understandings and meanings. The chapters clearly show the intersection of the materialisms-embodiment concepts and, therefore, could be placed in either section due to the fluid relationship of the materialisms-embodiment pair. Ageing in Everyday Life's strength is exploring the closely linked relationship between the concepts of materialities and embodiments. Is then dividing the readings into two separate parts (Part I: Materialities and Part II: Embodiments) at all necessary? Do these two parts impose divisions in the suggested fluidity of the materialities-embodiments continuum? Building upon the cover photo of a personified weathered boat, more visual representation - such as photo elicitation - could deepen the look at older adults seen in the work. Additionally, it would be helpful to see how the materialities concept works in non-western settings. And, could same relationships of older persons and things occur in a less materialistic culture where things may hold less attachment?

In conclusion, this edited volume is a vital contribution to the fields of gerontology and sociology, amongst others, as it reframes how we look at the everyday experience of ageing without reductionistic labels and lenses of health or activity. The chapters move beyond ageing materialities and embodiments, past surveillance and external markers of good embodiment - such as healthy or successful ageing. Including significant first-hand narratives keeps the older body heard and seen in the work.

\section{References}

Katz, S. (2018). Introduction. In Katz, S. (ed.), Ageing in Everyday Life. Materialities and Embodiments (pp. 1-21). Bristol: Policy Press.

Sawchuk, K. (2018). Afterword. Relational entanglements: Ageing, materialities and embodiments. In Katz, S. (ed.), Ageing in Everyday Life. Materialities and Embodiments (pp. 215-224). Bristol: Policy Press. 
